\title{
Nafion-Coated Bismuth Film and Nafion- Coated Mercury Film Electrodes for Anodic Stripping Voltammetry Combined On-Line with ICP-Mass Spectrometry
}

\author{
Grace X. Cao, Omar Jimenez, and Feimeng Zhou \\ Department of Chemistry and Biochemistry, California State University, Los Angeles, California, USA \\ Maotian Xu \\ College of Chemistry and Chemical Engineering, Central South University, Hunan, China
}

\begin{abstract}
Nafion-coated bismuth film electrodes (NCBFEs) and Nafion-coated mercury film electrodes (NCMFEs) were used to electrochemically preconcentrate metal analytes for subsequent analysis by inductively coupled plasma-mass spectrometry (ICP-MS). Either type of electrodes is part of a thin-layer electrochemical flow cell that is positioned upstream of a microconcentric nebulizer for the ICP-MS. Performances of these electrodes were compared in terms of the analytical "figures of merit" (e.g., dynamic ranges, reproducibility, hydrodynamic stability, and elimination of matrix effects detrimental to ICP-MS). The coupled technique (ASV-ICPMS) is found to possess a wide dynamic range (at least 4 to 5 orders of magnitude) and to be reproducible. Both electrodes are much more stable than the thin mercury film electrode (TMFE) traditionally used for ASV-ICP-MS, with the lifetime of the NCBFE exceeding $8 \mathrm{~h}$. Adopting these electrodes for ASV-ICP-MS overcomes the problems associated with a TMFE, the erosion of which decreases the sample throughput, affects the analysis precision, and contaminates conventional glass nebulizers and spray chambers of the spectrometer. The medium exchange procedure inherent in ASV is successfully implemented with a two-valve flow injection system for the accumulation of trace $\mathrm{Cd}^{2+}$ into the electrode from a certified seawater sample, followed by stripping $\mathrm{Cd}$ into a solution that is compatible to the ICP-MS operation. (J Am Soc Mass Spectrom 2006, 17, 945-952) (c) 2006 American Society for Mass Spectrometry
\end{abstract}

$\mathrm{A}$ nodic stripping voltammetry (ASV) combined on-line with inductively coupled plasma-mass spectrometry (ASV-ICP-MS) [1, 2] or the ICPatomic emission spectrometry variant (ASV-ICP-AES) [3] has emerged as a powerful analytical technique for enhanced trace metal analysis [4-6], elimination of detrimental ICP matrix effects [7-9], and studies of electrode reactions involving adsorption/desorption of metals or metal-containing species [10-12]. Various applications of this combination have been reviewed [1, 2]. Earlier work in this field centered on the use of a flow-through electrochemical cell housing a mercurycoated reticulated vitreous carbon (RVC) working electrode [7, 13-16], and demonstrated that amalgam-forming metal ions (e.g., $\mathrm{Cd}^{2+}, \mathrm{Cu}^{2+}, \mathrm{Pb}^{2+}$, etc.) could be analyzed with few matrix effects [7]. The elimination of

Published online May 15, 2006

Address reprint requests to Dr. F. Zhou, Department of Chemistry and Biochemistry, California State University, Los Angeles, CA 90032, USA. E-mail: fzhou@calstatela.edu both ICP spectroscopic and nonspectroscopic interferences [17-19] resulted from the use of a mediumexchange procedure of ASV [20], in which the flow cell can be thoroughly washed with an interference-free solution (e.g., dilute $\mathrm{HNO}_{3}$ or high-purity ammonium acetate buffer) before diverting the cell eluent for the subsequent voltammetric scan and ICP-MS analysis. The stripped metals elute in distinct sample plugs and can be easily monitored by time-resolved ICP-MS measurements. Elements whose oxidation states are indistinguishable by ICP-MS (e.g., As(III) and $\mathrm{Cr}(\mathrm{VI}))$ have also been deposited onto and stripped off the RVC electrode for subsequent ICP-MS analysis [14, 16].

In the past ten years or so, efforts were focused on the use of a thin-layer flow cell incorporating a disk electrode $[4,5,8-11,21]$. Such a cell was demonstrated to be more advantageous than the flow-through cell, because greater preconcentration efficiency, faster cell clean-up, and smaller sample consumption can all be realized. The thin-layer flow cell design has been successfully utilized by Van Berkel and coworkers and 
Zhou and coworkers for quantification of radionuclides $[8,9]$, heavy metals in complex sample media $[12,14,16$, $22]$, ion incorporation and ejection from organic and polymeric thin films [11, 23], and metal release from biomolecular adsorbates [10]. However, for analysis employing thin mercury film electrodes (TMFEs), a direct injection nebulizer (DIN) [24-26] had to be incorporated into the setup to alleviate the memory effect caused by mercury eroded into the sample introduction system $[4,8]$. The lower sample flow rates allowed by the DIN [24, 26] also help extend the lifetime of the TMFE, allowing a longer analyte preconcentration to be carried out. Unfortunately, even under such a favorable experimental condition and with a relatively thick mercury film (e.g., $100 \mathrm{~nm}$ [4], the electrode was still found to last for typically about $60 \mathrm{~min}$ or less. Such a drawback severely reduces the sample throughput and affects the precision of the ASV-ICP-MS measurements. Consequently, fresh mercury films need to be periodically deposited, generating large amounts of toxic wastes.

Previous reports have shown that polymeric membranes or thin film coatings, such as Nafion [27-30], enhance the mechanical stability of TMFEs and improve voltammetric signal-to-noise ratios [31]. We, therefore, envisioned that Nafion-coated mercury film electrodes (NCMFEs) should be a viable remedy for conventional mercury films used for ASV-ICP-MS. Recently, bismuth film electrodes (BFEs) have emerged as an environmentally friendly alternative to TMFEs for ASV [32-36]. BFEs and Nafion-coated BFEs (NCBFEs) retain many desirable features of mercury (e.g., high voltammetric signal intensity, well-resolved stripping peaks, and high hydrogen overpotentials [20], are efficient in enriching metal ions, and possess high hydrodynamic stability. These electrodes (BFEs, NCBFEs, and TMFEs) are more advantageous than other commonly used solid electrodes (e.g., $\mathrm{Pt}$ and $\mathrm{Au}$ ) for ASV in terms of preconcentration efficiency and reproducibility. Moreover, the high hydrogen overpotentials allow them to be used in acidic media that generally used for trace metal analysis. In this work, they are shown to allow samples to be continuously analyzed with good precision and accuracy for an extended period of time $(>5$ h). Moreover, samples present in matrices that are not compatible to the ICP-MS operational condition can be pretreated and preconcentrated before sensitive ICP-MS quantification.

\section{Experimental}

\section{Reagents and Chemicals}

All solution preparations were conducted in a clean room with deionized water from a water purification system (Simplicity Plus, Millipore S.A. 67120 Molsheim, France). All metal standard solutions were acquired from Aldrich Chemicals (Milwaukee, WI). Ammonia and acetic acid (HAc) used for preparing the $\mathrm{NH}_{4} \mathrm{Ac}-$
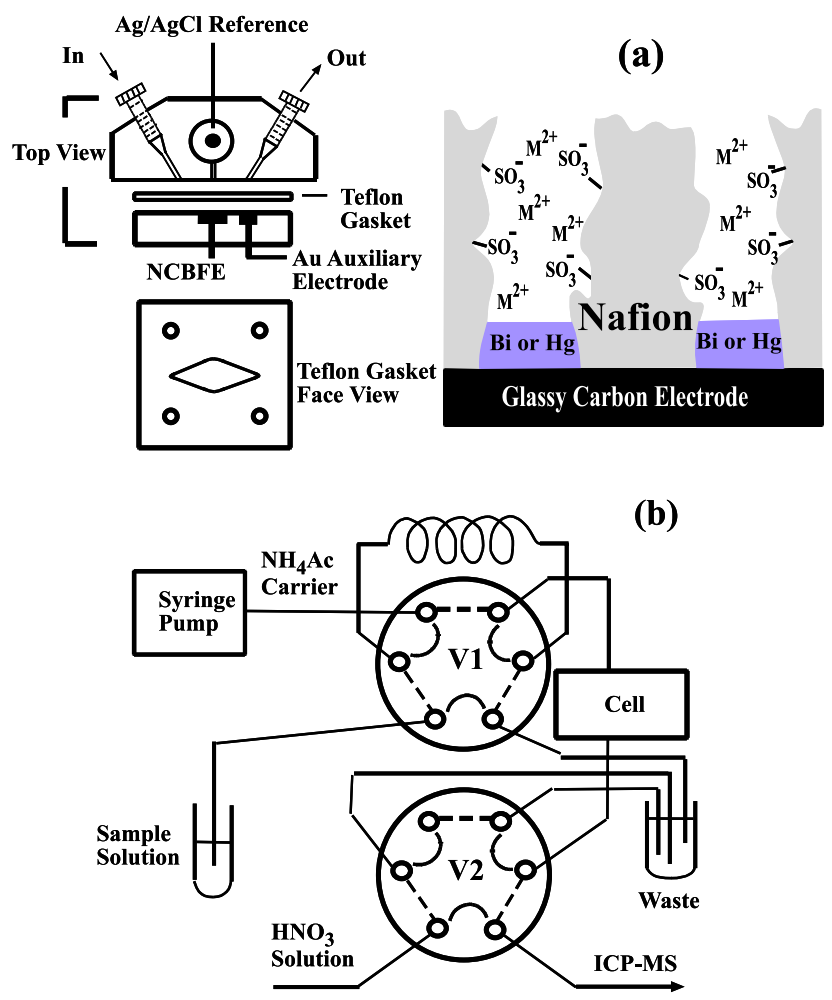

Figure 1. Schematic representations of the electrochemical flow cell (a) and a two-valve flow-injection system comprising the electrochemical flow cell for ASV-ICP-MS (b). A detailed side view of a Nafion-coated electrode. In (b), the solid lines that interconnect the ports on the valves represent the configuration for the sample preconcentration, and the dotted curves show that for anodic stripping and analyte elution to the ICP-MS. For clarity, the diagrams are not drawn to scale.

HAc buffer solution were doubly distilled from Vycor (GFS Chemicals, Powell, $\mathrm{OH}$ ). The $\mathrm{NH}_{4} \mathrm{Ac}-\mathrm{HAc}$ buffer solution was used to dilute all the metal standards. Nafion solutions $(5 \% \mathrm{wt} / \mathrm{vol}$ solution in a mixture of alcohol and water, Aldrich Chemicals) were used to prepare the electrodes. The $\mathrm{NH}_{4} \mathrm{Ac}-\mathrm{HAC}$ buffer allows the metal preconcentration step to be conducted in a slightly acidic solution ( $\mathrm{pH}=4.5)$, a condition commonly used for ASV at NCBFEs [31]. At the same time it does not produce interfering peaks in the ICP-mass spectra.

Cadmium present in a certified CASS-4 open ocean seawater reference (National Research Council, Ottawa, Canada) was used to assess the accuracy of and matrix effect elimination by the ASV-ICP-MS.

\section{Electrodes and Cells}

The flow cell has been slightly modified from the design used by Van Berkel [5,9] and coworkers and that for our previous work [4, 10] (Figure 1a). The Pt foil auxiliary electrode is replaced by a 3-mm-diameter gold disk electrode positioned next to the working electrode. Since the Pt foil could cause cell leakage, this change simplifies the operational procedure. It also facilitates a 
Table 1. Operating Parameters for the ICP-MS

\begin{tabular}{ll} 
RF Power & $1150 \mathrm{~W}$ \\
Nebulizer Gas Flow Rate & $0.95 \mathrm{~L} / \mathrm{min}$ \\
Auxiliary Gas Flow Rate & $1.20 \mathrm{~L} / \mathrm{min}$ \\
Plasma Gas Flow Rate & $15.00 \mathrm{~L} / \mathrm{min}$ \\
Analog Stage Voltage & $-1750 \mathrm{~V}$ \\
Pulse Stage Voltage & $1000 \mathrm{~V}$ \\
Lens Voltage $\left({ }^{115} / \mathrm{n}\right)$ & $9 \mathrm{~V}$ \\
Autolens & On \\
Sample Introduction System & $\mathrm{MCN}$ \\
Solution Uptake Rate & $100 \mu \mathrm{L} / \mathrm{min}$ \\
Solution Flow Mode & Flow Injection \\
Scan Mode & Peak Hopping \\
MCA Channels (points/mass) & 1 \\
Resolution & $0.7 \mathrm{amu}$ \\
Sweeps/Reading & 1 \\
Readings/Replicate & 4300 \\
Replicates & 1 \\
Dwell Time/amu & $350 \mathrm{~ms}$ \\
\hline
\end{tabular}

direct adoption of the commercially available stainless steel cell (Bioanalytical Systems Inc., West Lafayette, $\mathrm{IN})$, which houses the $\mathrm{Ag} / \mathrm{AgCl}$ reference electrode, into the experimental setup. For analysis of elements that are present in stainless steel (e.g., nickel and chromium), the cell surface can be coated with a 100-nm thick gold film with a 2-nm Cr underlayer. We found that such a gold film was rather robust and can last 4 to 5 days of experiments. Teflon gaskets (Figure 1a) of thicknesses of 51, 127, and $254 \mu \mathrm{m}$ (Bioanalytical Systems Inc.) were tested for the flow cell, with the 127 $\mu \mathrm{m}$-thick gasket deemed to be most suitable in terms of signal intensity and operational convenience. With this improved design, attaching the PEEK block embedding the glassy carbon working electrode and the Au auxiliary electrode (Figure 1a) to the cell body is convenient, cleaning the electrodes is fast, and obtaining undistorted voltammograms is routine.

\section{Instruments}

The ICP-MS was a double-quadrupole mass spectrometer (Elan-DRCe, Perkin-Elmer/Sciex Corp., Norwalk, $\mathrm{CT}$ ). A microconcentric nebulizer (MCN, CETAC Technologies Inc., Omaha, NE) was used in conjunction with a Scott-type spray chamber. The operating conditions for the MCN and the ICP-MS are listed in Table 1. Two six-port injection valves (Microneb 2000, CETAC) were connected to the inlet of the electrochemical flow cell (Figure 1). A dual-syringe pump (Kd Scientific Model 270, Holliston, MA) was used to deliver the $\mathrm{NH}_{4} \mathrm{Ac}-$ HAC buffer solution. A flow rate of $100 \mu \mathrm{L} / \mathrm{min}$ was chosen for the optimal MCN performance. Control of the electrode potentials was achieved using a $\mathrm{CHI}$ 615A electrochemical analyzer ( $\mathrm{CH}$ Instruments, Austin, TX).

\section{Procedures}

The glassy carbon electrode for forming the NCBFE or NCMFE was polished with diamond and alumina pastes (Buehler, Lake Bluff, IL) and sonicated in a water bath for $30 \mathrm{~s}$ to remove adherent polishing materials from the electrode surface. To prepare a NCBFE, the glassy carbon electrode was positioned upside down and $3 \mu \mathrm{L}$ of a $1 \%$ Nafion solution was pipeted onto the electrode surface and left in the ambient atmosphere to dry. Bismuth was then deposited through the Nafion matrix onto the bottoms of pores from a $0.5 \mathrm{M} \mathrm{HCl}$ solution containing $1.0 \mathrm{mM} \mathrm{Bi}^{3+}$ by applying $-0.6 \mathrm{~V}$ for $30 \mathrm{~s}$ in the flow system (i.e., on-line preparation). The NCMFEs were prepared off-line to avoid contamination of the flow cell with $\mathrm{Hg}^{2+}$ and to reduce toxic wastes. We found that NCMFEs prepared off-line generally produced higher and more reproducible ASV signals. To prepare NCMFEs, $3 \mu \mathrm{L}$ of $0.21 \%$ Nafion solution was first spread over the glassy carbon electrode. Immediately upon drying, $2 \mu \mathrm{L}$ dimethylformamide (DMF) was cast, and the electrode surface was dried under a heat gun $[28,29,37]$. Mercury films were deposited onto the bottoms of the pores in the Nafion film by holding the electrode at $-0.4 \mathrm{~V}$ in a $5.0 \mathrm{mM} \mathrm{Hg}{ }^{2+} / 1 \% \mathrm{HNO}_{3}$ solution for $300 \mathrm{~s}$.

Metals of interest were accumulated into these electrodes at a preset potential with deposition times ranging from 15 to $500 \mathrm{~s}$. Differential pulse voltammograms (DPVs) were recorded using a 50-ms pulse width and a $50-\mathrm{mV}$ amplitude. Metal ions that were stripped into the $\mathrm{NH}_{4} \mathrm{Ac}-\mathrm{HAC}$ carrier solution eluted out of the electrochemical flow cell to the ICP-MS. The elution profiles were monitored by ICP-MS using timeresolved acquisition in the peak-hopping mode. For determining metal analytes in complex matrices, sample solutions were introduced to the flow cell via valve 1 (cf. Figure $1 \mathrm{~b}$ ) and the cell content was directed to waste. At the same time, $1 \% \mathrm{HNO}_{3}$ solution flowed through valve 2 to the ICP-MS sample introduction system. The preconcentration step was followed by washing the cell with the $\mathrm{NH}_{4} \mathrm{Ac}-\mathrm{HAc}$ carrier solution for 60-150 s, switching valve 2 (Figure 1b) to the ICP-MS sample introduction system, and stripping metals into the $\mathrm{NH}_{4} \mathrm{Ac}-\mathrm{HAc}$ carrier solution.

\section{Results and Discussion}

\section{NCBFEs and NCMFEs for ASV-ICP-MS}

The principle behind ASV-ICP-MS has been reviewed and described previously $[1,2,8]$. Therefore, we provide in Figure 1a only a schematic illustration of the NCBFE or NCMFE structure. The as-prepared electrodes have an average Nafion film thickness of $0.68 \mu \mathrm{m}$ with a 8-nm thick Bi film or 100-nm thick Hg film [31]. The numerous pores in the Nafion matrix are about 5 $\mathrm{nm}$ in diameter [38]. The permselective cation exchange capability of the Nafion film should help accumulate the metal ions from dilute sample solutions, though the relatively high ionic strength of the carrier solution probably diminished the permselectivity to some extent (vide infra) $[37,39]$. As a result, mass transfer of the 
analyte within the Nafion matrix to metal films present at the bottom of the pores is not significantly restricted and the elution of the stripped ions is not retarded. By applying a suitable potential, metal ions can be reduced and deposited as metals into the Bi film or $\mathrm{Hg}$ film. After the cell has been washed with the carrier solution (cf. Figure 1b), metals deposited can be stripped for subsequent ICP-MS analysis. The dual-valve flow injection system was used by Pretty et al. for analysis of uranium at ultratrace levels using ASV-ICP-MS [9]. We replaced the gas displacement pump (GDP) with a dual-syringe pump in the same configuration as described in reference [9]. Using syringe pumps obviates the necessity of balancing the solution flows to the two valves and the flow rate calibration.

The possibility of using the NCBFE and NCMFE for multi-element analysis by ASV-ICP-MS is demonstrated in Figure 2. The dotted line curve in Figure 2a was acquired after holding a NCBFE electrode potential at $-1.200 \mathrm{~V}$ for $500 \mathrm{~s}$ to deposit various metal analytes, washing the cell, and initiating a differential pulse voltammetric (DPV) scan, whereas the solid line curve was obtained at a NCMFE electrode using $360 \mathrm{~s}$ for the preconcentration step. Figure $2 \mathrm{~b}$ and $\mathrm{c}$ are timeresolved ICP-MS responses corresponding to elutions of the metals stripped off the NCBFE and NCMFE electrodes, respectively. Oxidation of the preconcentrated $\mathrm{Tl}$ and $\mathrm{Cd}$ produced a single DPV peak $(-0.579 \mathrm{~V}$ at the NCBFE and $-0.545 \mathrm{~V}$ at the NCMFE), due to the overlapping stripping potentials of $\mathrm{Tl}$ and $\mathrm{Cd}$ [40]. As noted in our previous work based on a TMFE [4], ASV-ICP-MS relies on the mass-to-charge $(\mathrm{m} / \mathrm{z})$ ratios for elemental analysis. Thus distinctive elution peaks of the metal ions stripped of the electrode can be obtained (Figure $2 \mathrm{~b}$ and $\mathrm{c}$ ). Evidently, both the elution sequence and the peak widths in Figure $2 b$ and c correlate well with the oxidation potentials and peak widths of the DPV peaks (Figure 2a). Since the elution profile is analogous to that we observed at a TMFE [4], the Nafion coating does not appear to retard the elution of the metal ions to the ICP-MS. Thus, it is apparent that metal stripping and metal ion diffusion out of the Nafion film are much faster than the elution of the sample plugs from the cell to the ICP-MS. A major benefit offered by the MS detection is that complications inherent in voltammetry and from electrode processes are circumvented [2]. Therefore, as shown by previous studies [41, 42], MS can provide insight to electrochemical reactions. For example, the profiles in both Figure $2 \mathrm{~b}$ and $\mathrm{c}$ suggest that $\mathrm{Tl}^{+}$eluted before $\mathrm{Cd}^{2+}$, even though the standard reduction potential of $\mathrm{Tl}^{+}$at a $\mathrm{Hg}$ electrode $(-0.530 \mathrm{~V})$ is more positive than that of $\mathrm{Cd}^{2+}(-0.548 \mathrm{~V})$ [40]. To verify this observation, we conducted DPV measurements in a quiescent acetate buffer solution containing only $\mathrm{Cd}^{2+}$ or only $\mathrm{Tl}^{+}$ and found that $\mathrm{Tl}^{+}$was indeed easier to oxidize at these Nafion-coated electrodes (data not shown). Possibly the interaction between the Nafion coating and doubly charged cations (i.e., $\mathrm{Cd}^{2+}$ ) is slightly stronger than that
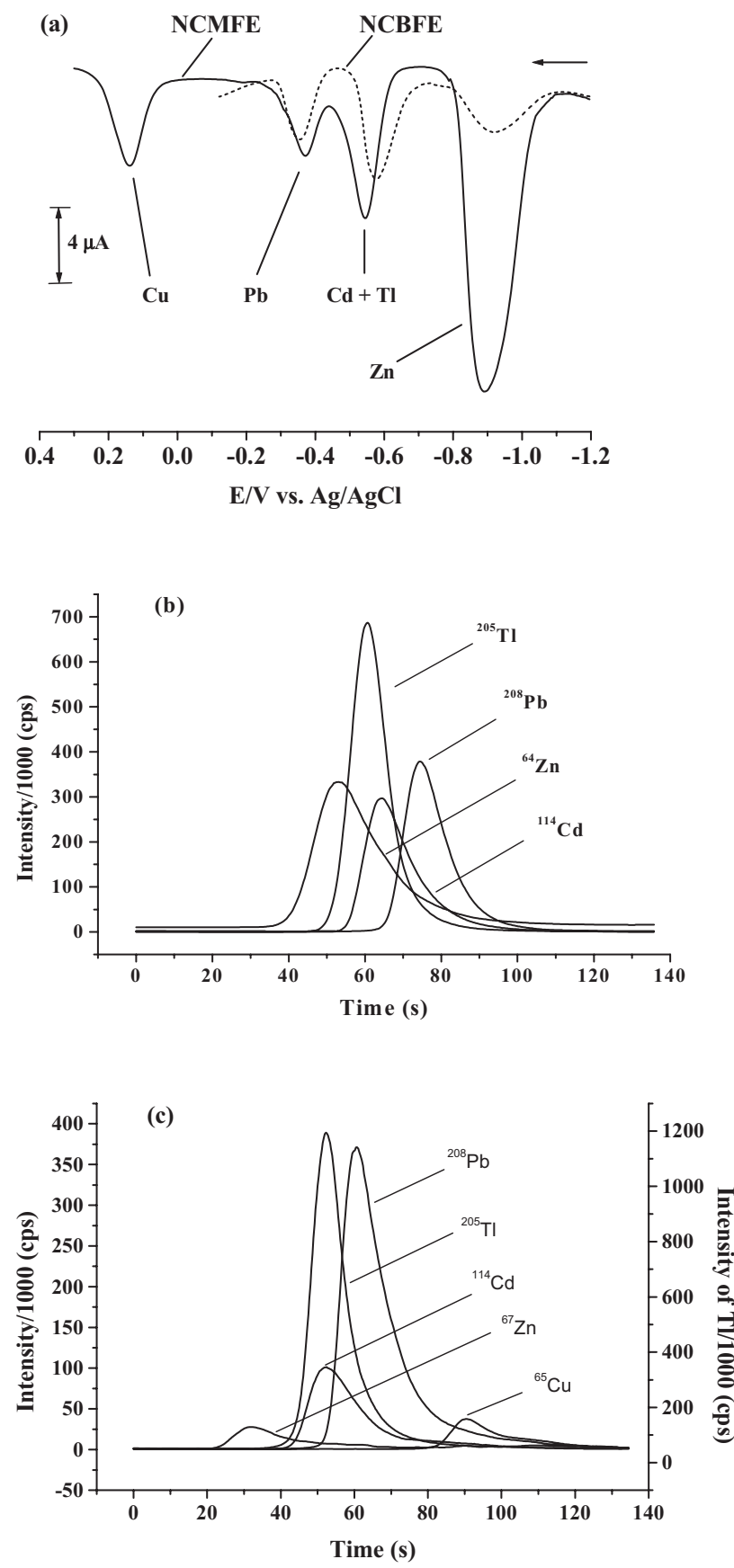

Figure 2. (a) Differential pulse voltammograms of $\mathrm{Zn}, \mathrm{Cd}, \mathrm{Tl}$, and $\mathrm{Pb}$ at a NCBFE (dotted line curve) and $\mathrm{Zn}, \mathrm{Cd}, \mathrm{Tl}, \mathrm{Pb}$, and $\mathrm{Cu}$ at a NCMFE (solid line curve) acquired in flowing streams of $\mathrm{NH}_{4} \mathrm{Ac}-\mathrm{HAc}$ buffer solutions $(\mathrm{pH}=4.5)$. The arrow indicates the scan direction. (b) Time-resolved ICP-MS responses to the eluted analytes upon ASV at the NCBFE [dotted line curve in (a)]. Concentrations of all the metal ions are $50 \mathrm{ppb}$ and the preconcentration time was $450 \mathrm{~s}$. (c) ICP-MS responses to the elution of metals resulted from ASV [solid line curve in (a)] at the NCMFE. Analyte concentration was $30 \mathrm{ppb}$ each. The ICP-MS signal for Tl was plotted on a different scale [right of (c)].

between the Nafion film and singly charged species (e.g., $\mathrm{Tl}^{+}$). Nevertheless, the retention is weak and the difference is only manifested when the stripping potentials between the two species are very close. 
Figure 2a shows that $\mathrm{Cu}$ can be stripped at $0.140 \mathrm{~V}$ at the NCMFE, a potential more positive than the $\mathrm{Bi}$ oxidation potential (at $0.112 \mathrm{~V}[44,45]$. Therefore, ASVICP-MS analysis of $\mathrm{Cu}$ should not be performed at a NCBFE. To confirm that metals stripped in their ionic forms are not retained by the Nafion film, we performed another potential scan and monitored the residual metal ion elution by ICP-MS. The intensity of the second set of elution peaks in the time-resolved ICP-MS responses are only 5 to $10 \%$ of that of the first set, suggesting that metals deposited can be quantitatively stripped. This is consistent with the aforementioned fact that the cation exchange (retaining) process in Nafion matrix is relatively slow [27]. The relatively high ionic strength used in this work $(0.1 \mathrm{M})$ might have negated the anionic property of Nafion film [37, 39] and thus decreased its tendency to electrostatically attract cationic species. The minimal retention by the Nafion coating results in the good linear relationship between the ICP-MS signals and the analyte concentrations (see below).

\section{Analytical Performance}

Dynamic range and reproducibility. With $\mathrm{Cd}^{2+}$ and $\mathrm{Pb}^{2+}$ standards as model analytes, we evaluated the dynamic ranges and reproducibility of ASV-ICP-MS at NCBFEs and NCMFEs (Figure 3). All the plots exhibited excellent linearity within the concentration ranges studied, with $R^{2}$ values exceeding 0.997 . The concentration ranges span 4 to 5 orders of magnitude. We did not attempt to measure concentrations in the ppm range, because relatively high analyte concentrations would not require sample preconcentration. Even for species present in media that tend to cause ICP-MS interferences, multiple dilutions can be carried out above the ICP-MS detection level to circumvent potential interferences. The error bars shown in the plots (with \%RSD ranging from 1 to $13 \%$ ) are relatively small, indicating that ASV-ICP-MS at either a NCBFE or a NCMFE is reproducible. The slopes of the $\mathrm{Cd}^{2+}$ calibration curves at these electrodes are smaller than those of the $\mathrm{Pb}^{2+}$ counterparts, implying that the ASV-ICP-MS sensitivity is higher for $\mathrm{Pb}$ than for $\mathrm{Cd}$. Such a trend can be attributed to the following three factors: (1) the $\mathrm{Pb}$ isotope monitored by ICP-MS $\left({ }^{208} \mathrm{~Pb}\right)$ is more abundant than the $\mathrm{Cd}$ isotope chosen for detection $\left({ }^{114} \mathrm{Cd}\right),(2) \mathrm{Pb}$ has higher ionization efficiency and less mass bias [19], and (3) $\mathrm{Pb}^{2+}$ is accumulated more effectively at these Nafion-coated electrodes. Another observation is that the slopes of the calibration curves for both metal ions at the NCMFE are greater than those at the NCBFE. This is conceivable given that metals can diffuse into $\mathrm{Hg}$ [18] more extensively than into the bismuth film.

Signal enhancement. We found that the ICP-MS signal intensity is proportional to the deposition (preconcentration) time. Figure 4 displays the ICP-MS peak intensities versus preconcentration times for $\mathrm{Pb}^{2+}$ and $\mathrm{Cd}^{2+}$
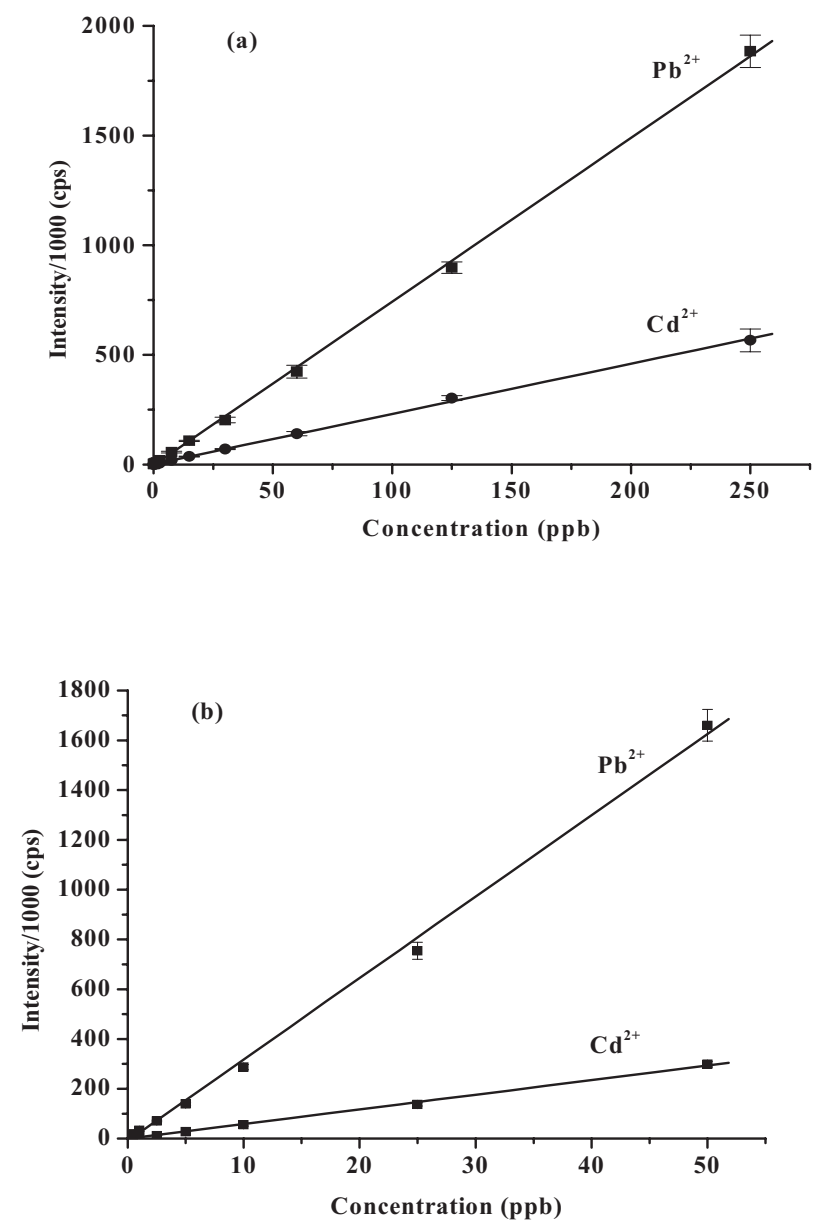

Figure 3. Calibration curves for $\mathrm{Pb}^{2+}$ and $\mathrm{Cd}^{2+}$ constructed from measuring a series of $\mathrm{Cd}^{2+}$ and $\mathrm{Pb}^{2+}$ standard solutions at a NCBFE (a) and a NCMFE (b) using ASV-ICP-MS. The preconcentration time was $500 \mathrm{~s}$ and the concentrations measured at the NCBFE were $0.013,0.025,0.050,0.15,0.30,0.60,1.3,2.5,7.5,15,30$, 60,125 , and $250 \mathrm{ppb}$ for $\mathrm{Cd}^{2+}$ and $\mathrm{Pb}^{2+}$. At the NCMFE, 0.005, $0.025,0.050,0.10,0.25,0.50,1.0,2.5,5.0,10,25$, and $50 \mathrm{ppb}$ of $\mathrm{Cd}^{2+}$ and $\mathrm{Pb}^{2+}$ were analyzed after a 360 -s preconcentration. The error bars were calculated from at least three replicates.

at a NCBFE (Figure 4a) and a NCMFE (Figure 4b). $\mathrm{R}^{2}$ values of these plots range from 0.995 to 0.998 . At both types of electrodes, the $\mathrm{Pb}^{2+}$ plot has a steeper slope than the $\mathrm{Cd}^{2+}$ plot. This is consistent with the three factors mentioned in the previous section to explain the difference in the calibration curve slopes. The extent of signal enhancement was estimated by comparing the ICP-MS peak intensities upon electrochemical preconcentration to signals obtained from directly nebulizing the sample solution. A 360-s preconcentration of a sample containing $30 \mathrm{ppb} \mathrm{Pb}^{2+}$ and $30 \mathrm{ppb} \mathrm{Cd}^{2+}$ at a NCMFE resulted in signal enhancements by factors of 8.0 and 2.8 times, respectively. The enhancement factor for $\mathrm{Cd}^{2+}$ is about 3 times lower than what we achieved at TMFEs used in conjunction with a DIN [4]. At least three factors contribute to this decrease: (1) the Nafion coating reduces the total volume of electroactive materials for analyte preconcentration (cf. Figure 1a, which shows that only the pores are partially filled with Bi or 

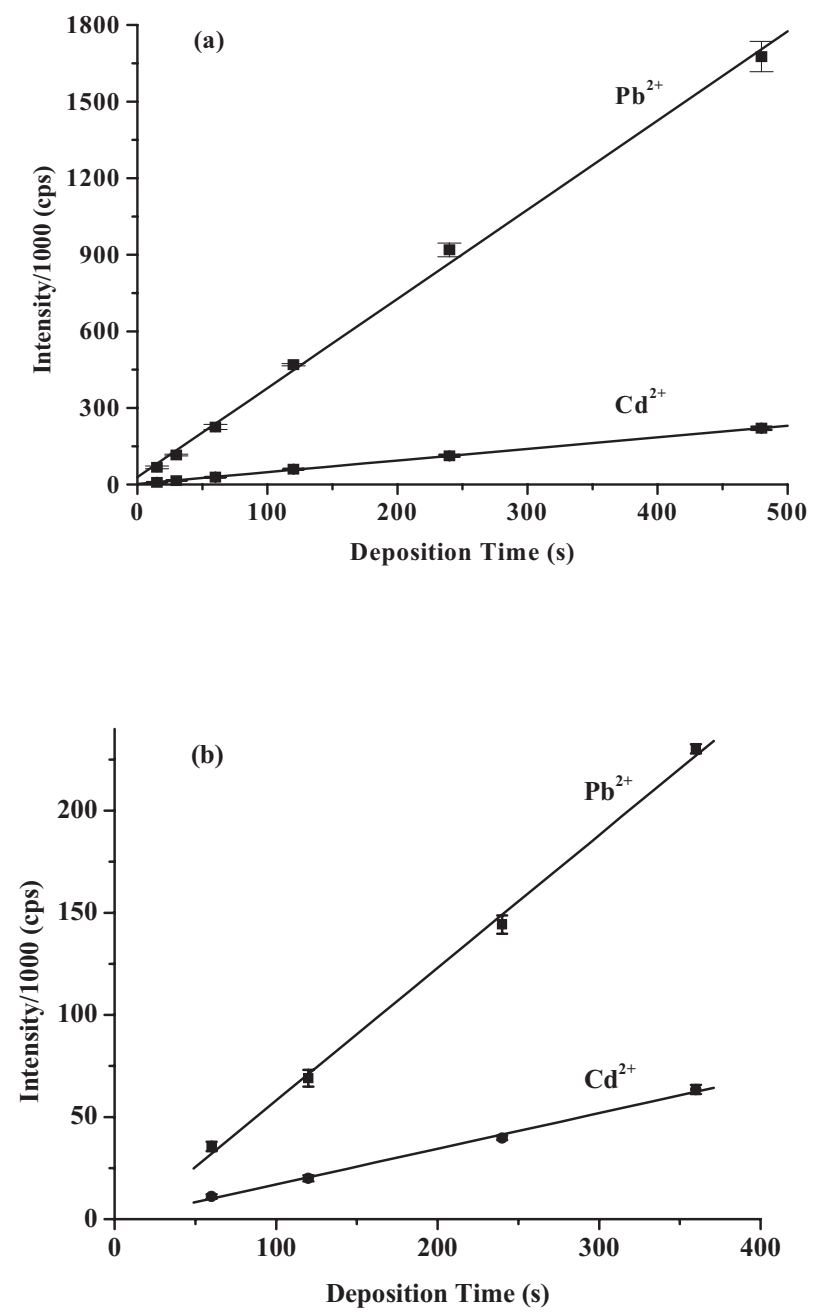

Figure 4. ICP-MS signal intensities for $\mathrm{Pb}^{2+}$ and $\mathrm{Cd}^{2+}$ versus deposition times at a NCBFE (a) and a NCMFE (b). Data were acquired using $56 \mathrm{ppb} \mathrm{Cd}^{2+}$ and $126 \mathrm{ppb} \mathrm{Pb}^{2+}$ for the NCBFE (a) and $30 \mathrm{ppb} \mathrm{Cd}^{2+}$ and $30 \mathrm{ppb} \mathrm{Pb}^{2+}$ for the NCMFE (b).

$\mathrm{Hg}),(2)$ the slower flow rate used for the DIN work (45 to $55 \mu \mathrm{L} / \mathrm{min}[4]$ increases the analyte deposition efficiency [5], and (3) as aforementioned, the preconcentration efficiency of $\mathrm{Cd}^{2+}$ at the Nafion-coated electrodes is relatively low (e.g., lower than $\mathrm{Pb}^{2+}$ ).

Durability of the NCBFEs and NCMFEs. To assess whether NCBFEs and/or NCMFEs are suitable for routine ASV-ICP-MS analysis, we examined their hydrodynamic stabilities by performing continuous measurements of a sample solution over an extended period of time. As clearly shown in Figure 5, at a NCBFE, no appreciable ICP-MS signal degradation was observed even after $4.5 \mathrm{~h}$. In fact, a single NCBFE is sufficient for a day's analysis $(\sim 8 \mathrm{~h})$. The ICP-MS signal intensity at a NCMFE, on the other hand, began to decay seriously after about $4 \mathrm{~h}$. Despite a somewhat shorter lifetime, a NCMFE is still substantially more stable than a TMFE (a 100-nm thick $\mathrm{Hg}$ film at the same flow rate lasted for less than $0.5 \mathrm{~h}$ ). Another attractive feature of NCBFEs
$[31,32,34,35]$ is that degassing of the analyte samples, as required for ASV at a TMFE, is not necessary. The improvement of the hydrodynamic stability of the bismuth or mercury thin film by the Nafion coating has been noted in the literature [27-29]. Such stability is in contrast with the erosion of the $\mathrm{Hg}$ film, which rapidly exposes the underlying glassy carbon surface. The exposed carbon surface can create hydrogen bubbles at a negative potential, affecting analyte deposition and analytical precision. This problem exacerbates for metals whose reduction potentials are very negative (e.g., $\mathrm{Zn}$ ). Based on these features, clearly the sample throughput of ASV-ICP-MS at NCBFEs or NCMFEs is greatly improved.

Accuracy and matrix effect elimination. To gauge the accuracy of ASV-ICP-MS and the effectiveness of the medium-exchange procedure for on-line sample cleanup, we measured $\mathrm{Cd}^{2+}$ in a certified seawater sample. The high salinity in the seawater sample could clog the fine capillary tubing in the MCN and/or the ICP-MS skimmer/sampler cones, and create polyatomic interferences (e.g., ${ }^{23} \mathrm{Na}^{40} \mathrm{Ar}^{+}$versus ${ }^{63} \mathrm{Cu}^{+}$[13]. Furthermore, ICP-MS signal suppression or even enhancement could also occur when a large number of ions are introduced to the MS system [18, 45]. As a consequence, continuous and accurate measurements of such samples cannot be achieved without significant sample dilution. Unfortunately, for species present at ultratrace levels, a significant dilution may decrease the analyte concentration below the ICP-MS detection limit. This point is reflected by the fact that curve I in Figure 6, which was obtained by injecting the certified seawater sample (diluted 30 times with the $\mathrm{NH}_{4} \mathrm{Ac}-\mathrm{HAc}$ buffer solution), is indistinguishable from the carrier signal. With ASV-ICP-MS, Cd can be accumulated into the NCBFE or NCMFE directly from the undiluted sample and stripped back into a solution that is benign to the ICP-MS operation (curve II). Furthermore, the method of standard addition can be readily implemented (curve

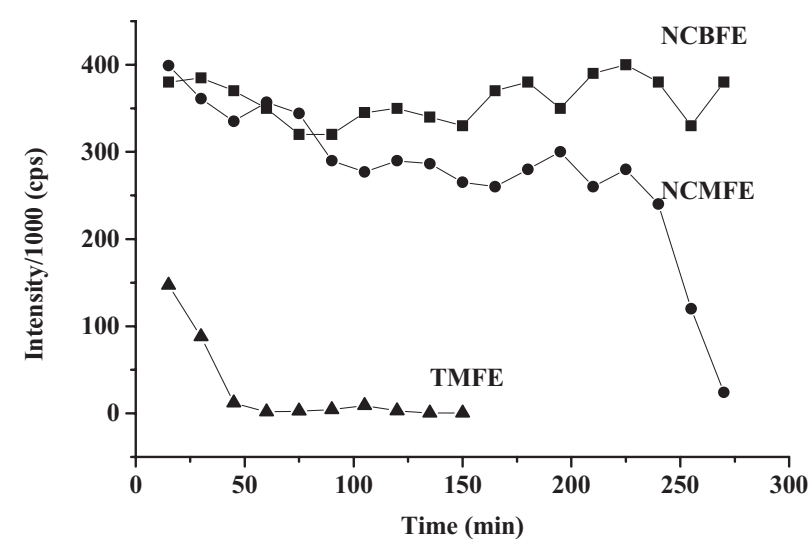

Figure 5. Hydrodynamic stabilities of a NCBFE, a NCMFE, and a TMFE demonstrated by plotting the ASV-ICP-MS signal intensities of $\mathrm{Pb}^{2+}(30 \mathrm{ppb})$ versus the total number of analyses. Each analysis was conducted at a 15-min increment. 


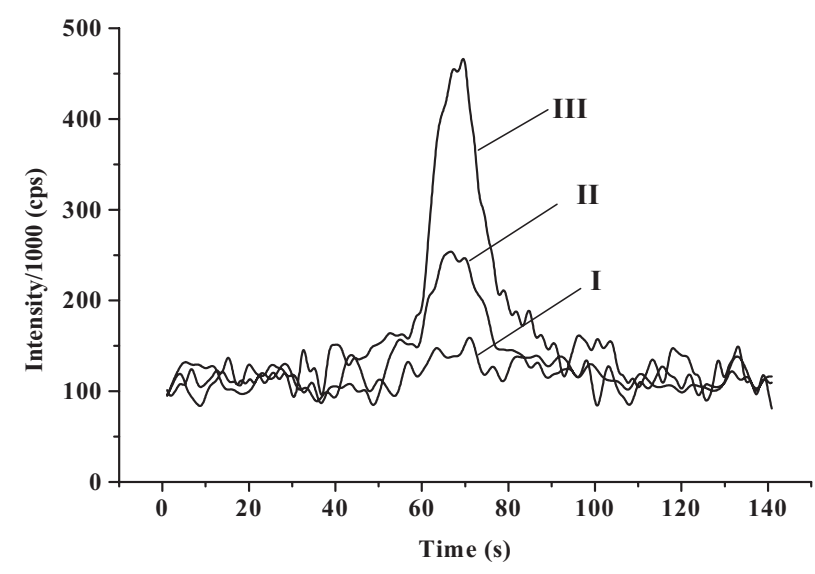

Figure 6. Time-resolved ICP-MS responses to $\mathrm{Cd}^{2+}$ in a certified seawater sample that has been diluted 30 times with a $0.1 \mathrm{M}$ $\mathrm{NH}_{4}$ Ac-HAc solution and injected directly to the ICP-MS (Curve I), and $\mathrm{Cd}^{2+}$ from the undiluted sample upon a 360-s preconcentration, followed by washing the flow cell with and stripping metal analytes into the $\mathrm{NH}_{4} \mathrm{Ac}-\mathrm{HAc}$ carrier solution (Curve II). Curve III: same as Curve II except that the sample was spiked with a 70 ppt $\mathrm{Cd}^{2+}$ standard.

III). With ASV-ICP-MS, we determined $\mathrm{Cd}^{2+}$ in this sample to be $36.2 \pm 2.2 \mathrm{ppt}$ at a NCBFE and $32.4 \pm 1.9$ ppt at a NCMFE, both of which are in reasonable agreement with the certified value $(26 \pm 3 \mathrm{ppt})$. The efficacy of the present work for matrix effect elimination compares well to that of our previous work based on a TMFE [4]. However, the environmentally friendly $\mathrm{Bi}$ and the prevention of rapid erosion of thin mercury film by the Nafion coating facilitate the adoption of the easy-to-use MCN and the durability of these electrodes makes the present methodology attractive for highthroughput environmental analysis.

\section{Conclusion}

NCBFEs and NCMFEs are both more desirable than TMFEs for heavy metal analysis based on ASV-ICP-MS. We demonstrate that ASV-ICP-MS utilizing these electrodes preserves the excellent "figures of merit" of ICP-MS (e.g., reproducibility, dynamic range, sensitivity, and accuracy), and at the same time, provides an appealing route for enhancing small signals associated with extremely low analyte concentrations and for eliminating detrimental ICP-MS matrix effects. While both electrodes are suitable for ASV-ICP-MS using conventional nebulizers/spray chambers, they complement each other in certain aspects. For example, NCMFEs enable $\mathrm{Cu}^{2+}$ to be analyzed at a trace level, whereas NCBFEs are nontoxic and do not require sample solutions to be deaerated. The hydrodynamic stabilities of these electrodes are much greater than those of TMFEs in flowing solution streams, and consequently replicate ASV-ICP-MS measurements can be performed without repeatedly making new mercury films. This drastically reduces the generation of toxic wastes and enhances sample throughputs. Finally, stabilized mercury films are more compatible with easy-to-implement nebulizers/spray chambers. The present work is thus complementary to ultrasensitive ASV-ICP-MS analyses performed with highly efficient nebulizers (e.g., DIN). Though not explored, we should add that the Nafion coating should be able to effectively exclude dissolved organic matters in environmental samples [27] (e.g., estuarine waters [37]. Another important aspect is that ICP-MS responses can be correlated with the voltammetric data to shed light on electrode processes. Since MS techniques are generally more sensitive and selective, we anticipate that ICP-MS and other MS techniques [22, 41-43] will be viable for on-line identification and quantification of various electrogenerated products.

\section{Acknowledgments}

FZ thanks Dr. Gary J. Van Berkel for the supervision and training on mass spectrometry and flow injection analysis during his postdoctoral stint at the Oak Ridge National Laboratory (19931995). The authors gratefully acknowledge partial support of this work by a NIH-SCORE subproject (GM-08,101), the NIH-RIMI Program (P20 MD001824-01) at California State University, Los Angeles, a NIH-AREA grant (GM-R15-63,530-10), and National Natural Science Foundation of China (no. 20225517). FZ also thanks Dr. Zed Mason and Dr. Grady Hanrahan for their kind help.

\section{References}

1. Zhou, F. Stripping Analysis Combined On-Line with Inductively Coupled Plasma-Atomic Emission Spectrometry and with Inductively Coupled Plasma-Mass Spectrometry. Electroanalysis 1996, 8, 855-861.

2. Zhou, F. Electrochemistry Combined On-Line with Atomic Mass Spectrometry and Related Techniques for Trace Metal Analysis and Studies of Electrode Reactions. Trends Anal. Chem. 2005, 24, 218-227.

3. Long, S. E.; Snook, R. D. Electrochemical Preconcentration Technique for Use with Inductively Coupled Plasma Atomic Emission Spectrometry. Analyst 1983, 108, 1331-1338.

4. Baca, A. J.; De La Ree, A. B.; Zhou, F.; Mason, A. Z. Anodic Stripping Voltammetry Combined On-Line with Inductively Coupled Plasma via a Direct-Injection High-Efficiency Nebulizer. Anal. Chem. 2003, 75, 2507-2511.

5. Pretty, J. R.; Duckworth, D. C.; Van Berkel, G. J. Anodic Stripping Voltammetry Coupled On-Line with Inductively Coupled Plasma Mass Spectrometry: Optimization of a Thin-Layer Flow Cell System for Analyte Signal Enhancement. Anal. Chem. 1997, 69, 3544-3551.

6. Yuan, D.; Wang, X.; Yang, P.; Huang, B. On-Line Electrolytic Dissolution of Alloys and Multielement Determination by Inductively Coupled Plasma Atomic Emission Spectrometry. Anal. Chim. Acta 1991, 251, 187-190.

7. Pretty, J. R.; Evans, E. H.; Blubaugh, E. A.; Shen, W. L.; Caruso, J. A.; Davidson, T. M. Minimization of Sample Matrix Effects and Signal Enhancement for Trace Analytes Using Anodic Stripping Voltammetry with Detection by Inductively Coupled Plasma Atomic Emission Spectrometry and Inductively Coupled Plasma Mass Spectrometry. J. Anal. At. Spectrom. 1990, 5, 437-443.

8. Zhou, F.; Van Berkel, G. J.; Morton, S. J.; Duckworth, D. C.; Adeneiyi, W. K.; Keller, J. M. In Applications of Inductively Coupled Plasma-Mass Spectrometry to Radionuclide Determinations; Morrow, R. W.; Crain, J. S., Eds; American Society for Testing and Materials: West Conshohocken, 1995; pp 82-98.

9. Pretty, J. R.; Duckworth, D. C.; Van Berkel, G. J. Electrochemical Sample Pretreatment Coupled On-Line with ICP-MS: Analysis of Uranium Using an Anodically Conditioned Glassy Carbon Working Electrode. Anal. Chem. 1998, 70, 1141-1148.

10. Baca, A. J.; Garcia, Y.; Briseno, A. L.; Zhou, F. Quantification of Metals Released by Metallothionein Adsorbates at Mercury Film Electrodes by Electrochemical ICP-Atomic Emission Spectrometry. J. Electroanal. Chem. 2001, 513, 25-35.

11. Briseno, A. L.; Baca, A. J.; Zhou, Q.; Lai, R.; Zhou, F. Quantification of Dopant Ions in Polypyrrole Films with Electrochemical ICP-Atomic Emission Spectrometry and Comparison to Electrochemical Quartz Crystal Microbalance Studies. Anal. Chim. Acta 2001, 441, 123-124. 
12. Pretty, J. R.; Van Berkel, G. J.; Duckworth, D. C. Adsorptive Stripping Voltammetry as a Sample Pretreatment Method for Trace Uranium Determinations by Inductively Coupled Plasma Mass Spectrometry. Int. J. Mass Spectrom. Ion Processes 1998, 178, 51-63.

13. Pretty, J. R.; Blubaugh, E. A.; Caruso, J. A. Determination of Copper and Cadmium Using an On-Line Anodic Stripping Voltammetry Flow Cell with Detection by Inductively Coupled Plasma Mass Spectrometry. J. Anal. At. Spectrom. 1992, 7, 1131-1137.

14. Pretty, J. R.; Blubaugh, E. A.; Caruso, J. A. Determination of Arsenic(III) and Selenium(IV) Using an On-Line Anodic Stripping Voltammetry Flow Cell with Detection by Inductively Coupled Plasma Atomic Emission Spectrometry and Inductively Coupled Plasma Mass Spectrometry. Anal. Chem. 1993, 65, 3396-3404.

15. Pretty, J. R.; Caruso, J. A. Signal Enhancement of Lead and Thallium in Inductively Coupled Plasma Atomic Emission Spectrometry Using On-Line Anodic Stripping Voltammetry. J. Anal. At. Spectrom. 1993, 8, 545-550.

16. Pretty, J. R.; Blubaugh, E. A.; Caruso, J. A.; Davidson, T. M. Determination of Chromium(VI) and Vanadium(V) Using an On-Line Anodic Stripping Voltammetry Flow Cell with Detection by Inductively Coupled Plasma Mass Spectrometry. Anal. Chem. 1994, 66, 1540-1547.

17. Montaser, A.; Golightly, E. W. Inductively Coupled Plasma in Atomic Spectrometry; VCH Publishers: New York, 1992, pp 300-335.

18. Olesik, J. W. In Inorganic Mass Spectrometry; Barshick, C. M.; Duckworth, D. C.; Smith, D. H., Eds; Marcel Dekker: New York, 2000; pp 91-92.

19. Taylor, H. E. In Inductively Coupled Plasma Mass Spectrometry: Practices and Techniques; Academic Press: San Diego, 2001; pp 25-27.

20. Wang, J. Stripping Analysis: Principles, Instrumentation, and Applications; VCH: Deerfield, FL, 1985, pp 48-51.

21. Pretty, J. R.; Van Berkel, G. J. Electrochemical Sample Pretreatment Coupled On-Line with Electrospray Mass Spectrometry for Enhanced Elemental Analysis. Rapid Commun. Mass Spectrom. 1998, 12, 1644-1652.

22. Zhou, F.; Van Berkel, G. J. Electrochemistry Combined On-Line with Electrospray Mass Spectrometry. Anal. Chem. 1995, 67, 3643-3649.

23. Kertesz, V.; Dunn, N. M.; Van Berkel, G. J. ElectrochemistryElectrospray-Mass Spectrometry Study of Cesium Uptake in Nickel Hexacyanoferrate Films. Electrochim. Acta 2002, 47, 1035-1042.

24. Wiederin, D. R.; Smith, F. G.; Houk, R. S. Direct Injection Nebulization for Inductively Coupled Plasma Mass Spectrometry. Anal Chem. 1991, $63,219-225$.

25. McLean, J. A.; Acon, B. W.; Montaser, A.; Singh, J.; Pritchard, D. L.; Patierno, S. R. The Determination of Cr in Human Lung Fibroblast Cells Using a Large Bore Direct Injection High Efficiency Nebulizer with Inductively Coupled Plasma Mass Spectrometry. Appl. Spectrosc. 2000, $54,659-663$.

26. Minnich, M. G.; Montaser, A. Direct Injection High Efficiency Nebulization in Inductively Coupled Plasma Mass Spectrometry Under Normal and Cool Plasma Conditions. Appl. Spectrosc. 2000, 54, 1261-1269.

27. Economou, A.; Fielden, P. R. Mercury Film Electrodes: Developments, Trends, and Potentialities for Electroanalysis. Analyst 2003, 128, 205212.

28. Hoyer, B.; Florence, T. M. Application of Polymer-Coated Glassy Carbon Electrodes to the Direct Determination of Trace Metals in Body
Fluids by Anodic Stripping Voltammetry. Anal. Chem. 1987, 59, 2839-2842.

29. Hoyer, B.; Jensen, N. Signal Stability of Nafion-Coated Thin Mercury Film Electrodes for Stripping Voltammetry. Talanta 1994, 41, 449-453.

30. Matysik, F. M.; Matysik, S.; Oliveira-Brett, A. M.; Brett, C. M. A. Ultrasound-enhanced Anodic Stripping Voltammetry Using Perfluorosulfonated Ionomer-Coated Mercury Thin-Film Electrodes. Anal. Chem. $1997,69,1651-1656$.

31. Kefala, G.; Economou, A.; Voulgaropoulos, A. A Study of NafionCoated Bismuth-Film Electrodes for the Determination of Trace Metals by Anodic Stripping Voltammetry. Analyst 2004, 129, 1-20.

32. Wang, J.; Lu, J.; Hocevar, S. B.; Farias, P. A. M. Bismuth-Coated Carbon Electrodes for Anodic Stripping Voltammetry. Anal. Chem. 2000, 72, 3218-3222.

33. Wang, J.; Deo, R. P.; Thongngamdee, S.; Ogorevc, B. Effect of SurfaceActive Compounds on the Stripping Voltammetric Response of Bismuth Film Electrodes. Electroanalysis 2001, 13, 1153-1156.

34. Wang, J.; Lu, J.; Kirgoz, U. A.; Hocevar, S. B.; Ogorevc, B. Insights into the Anodic Stripping Voltammetric Behavior of Bismuth Film Electrodes. Anal. Chim. Acta 2001, 434, 29-34.

35. Kefala, G.; Economou, A.; Voulgaropoulos, A.; Sofoniou, M. A Study of Bismuth-Film Electrodes for the Detection of Trace Metals by Anodic Stripping Voltammetry and Their Application to the Determination of $\mathrm{Pb}$ and $\mathrm{Zn}$ in Tap Water and Human Hair. Talanta 2003, 61, 603-610.

36. Banks, C. E.; Kruusma, J.; Hyde, M. E.; Salimi, A.; Compton, R. G. Sonoelectroanalysis: Investigation of Bismuth-Film-Modified Glassy Carbon Electrodes. Anal. Bioanal. Chem. 2004, 379, 277-282.

37. Hurst, M. P.; Bruland, K. W. The Use of Nafion-Coated Thin Mercury Film Electrodes for the Determination of the Dissolved Copper Speciation in Estuarine Water. Anal. Chim. Acta 2005, 546, 68-78.

38. Seen, A. J. Nafion: An Excellent Support for Metal-complex Catalysts. J. Mol. Catal. 2001, 177, 105-112.

39. Guy, R. D.; Namaratne, S. Nafion-Coated Mercury-Coated Glassy Carbon Electrodes for Metals Analysis and Speciation. Can. J. Chem. 1987, 65, 1133-1138.

40. Bard, A. J.; Faulkner, L. R. Electrochemical Methods. Fundamentals and Applications; John Wiley and Sons: New York, 2001, p 808.

41. Karst, U. Electrochemistry/Mass Spectrometry (EC/MS)—A New Tool to Study Drug Metabolism and Reaction Mechanisms. Angew. Chem. Int. Ed. 2004, 43, 2476-2478

42. Volk, K. J.; Yost, R. A.; Brajter-Toth, A. Electrochemistry On-Line with Mass Spectrometry. Insight into Biological Redox Reactions. Anal. Chem. 1992, 64, 21A-33A.

43. Volk, K. J.; Yost, R. A.; Brajter-Toth, A. On-Line Electrochemistry/ Thermospray/Tandem Mass Spectrometry as a New Approach to the Study of Redox Reactions: The Oxidation of Uric Acid. Anal. Chem. 1989, 61, 1709-1717.

44. Harris, D. C. Quantitative Chemical Analysis; W. H. Freeman and Company: New York, 2003, pp AP23-AP24.

45. Skoog, D. A.; Holler, F. J.; Nieman, T. A. Principles of Instrumental Analysis; Saunders College Publishing: Philadelphia, 1992, pp A45A46. 